\title{
Quantitative Methods for Comparisons between Velocity Encoded MR-Measurements and Finite Element Modeling in Phantom Models
}

\author{
Frieke M.A. Box ${ }^{1}$, Marcel C.M. Rutten ${ }^{3}$, Mark A. van Buchem ${ }^{2}$, Joost \\ Doornbos $^{2}$, Rob J. van der Geest ${ }^{1}$, Patrick J.H. de Koning ${ }^{1}$, Jorrit Schaap ${ }^{1}$, \\ Frans N. van de Vosse $^{3}$, and Johan H.C. Reiber ${ }^{1}$ \\ 1 Division of Image Processing, Department of Radiology, Leiden University Medical \\ Center, Leiden, the Netherlands \\ 2 Department of Radiology, Leiden University Medical Center, Leiden, the \\ Netherlands \\ ${ }^{3}$ Department of Biomedical Engineering, Eindhoven University of Technology, \\ Eindhoven, the Netherlands
}

\begin{abstract}
Wall Shear Stress is a key factor in the development of atherosclerosis. To assess the WSS in-vivo, velocity encoded MRI is combined with geometry measurements by 3D MR-Angiography (MRA) and with blood flow calculations using the Finite Element Method (FEM). The 3D geometry extracted from the MRA data was converted to a mesh suitable for FEM calculations. Aiming at in-vivo studies the goal of this study was to quantify the differences between FEM calculations and MRI measurements. Two phantoms, a curved tube and a carotid bifurcation model were used. The geometry and the time-dependent flow-rate (measured by MRI) formed input for the FEM calculations. For good data quality, $2 \mathrm{D}$ velocity profiles were analyzed further by the KolmogorovSmirnov method. For the curved tube calculations and measurements matched well ( prob $_{K S}$ approximately above 0.20 ). The carotid needs further investigation in segmentation and simulation to obtain similar results. It can be concluded that the error-analysis performs reliably.
\end{abstract}

\section{Introduction}

It is known that a correlation exists between the presence of atherosclerosis and the local Wall Shear Stresses (WSS) in arteries [2]. The WSS is defined as the mechanical frictional force exerted on the vessel wall by the flowing blood. The WSS $\tau_{w}$ is defined as wall shear rate $\dot{\gamma}$ multiplied by the dynamic viscosity $\eta$ :

$$
\tau_{w}=\eta \dot{\gamma}
$$

Near the wall $\dot{\gamma}$ may be expressed as the velocity gradient with respect to the outward normal $\boldsymbol{n}$ of the wall:

$$
\tau_{w}=\eta \frac{\partial v}{\partial n}
$$

P.M.A. Sloot et al. (Eds.): ICCS 2002, LNCS 2331, pp. 255-264, 2002.

(C) Springer-Verlag Berlin Heidelberg 2002 
with $v$ being the fluid velocity. To be able to assess the local WSS distribution from MRI-images of arteries a good approximation of the local velocity profiles is required. One of the major drawbacks of MRI-velocity-data is the relatively low resolution and the unknown error distribution (noise). To get around this problem, finite element(FEM) calculations may be used and compared with actual MRI-measurements. Crucial for precise and reliable measurements, however, is a thorough error analysis. Therefore, the goal of our study was to determine in a quantitative manner the correspondences and differences between actually measured time-dependent flow rates (flow $(\mathrm{t})$ ) and velocity profiles by MRI, with the corresponding flow $(\mathrm{t})$ and velocity profiles derived from FEM calculations. The Kolmogorov-Smirnov method was applied to quantify the similarities between the 2D velocity profiles for FEM calculations and velocity encoded MRI measurements. Materials used for this study were two phantom models, one being a $90^{\circ}$ curved tube and the other a carotid bifurcation. The curved tube was analyzed at three positions, i.e. at the inflow, in the middle section, and at the outflow. Data for the carotid phantom was assessed at two positions, i.e. at the entrance and just behind the bifurcation.

\section{Materials and Methods}

Two PMMA (polymethyl methacrylate) phantom models, one of a curved tube (diameter of $8 \mathrm{~mm}$ ) and one of a carotid artery (inflow diameter of $8 \mathrm{~mm}$, outflow diameters of 5.6 and $4.6 \mathrm{~mm}$, for the internal carotid and external carotid arteries respectively), were connected to a MRI compatible pump (Shelley Medical Imaging Technologies), which can deliver an adjustable (pulsatile) flow profile flow $(\mathrm{t})$. The blood emulating fluid is a Newtonian fluid with a viscosity of $2.5 \mathrm{mPa}$.s (Shelley) and MRI-compatible. The phantoms were connected to a straight and fixed tube with a length of $110 \mathrm{~cm}$; as a result the inflow velocity profile is known and can be described analytically (Womersley-profile) $[5,13]$. The phantoms were scanned and processed with a $1.5 \mathrm{~T}$ MR system (Philips Medical Systems) using a standard knee-coil in two ways:

1. The geometry of each phantom was obtained by means of a MR Angiographic acquisition protocol. The tubes were divided into 100 slices with a slice thickness of $1 \mathrm{~mm}$, a TE/TR $6.8 / 21 \mathrm{~ms}$, a field-of-view (FOV) of $256 \mathrm{~mm}$, and a scan matrix of $512 \times 512$ pixels.

2. At different positions along the carotid- and curved tube model, the velocity and flow were assessed in a plane perpendicular to the major flow direction. Velocity encoded data were obtained by means of a gradient echo phase contrast imaging procedure. Triggering was applied during the acquisition and the simulated cardiac cycle was subdivided into 25 equidistant phases. The imaging parameters were: TE/TR $11.2 / 18.44 \mathrm{~ms}$, flip angle 15 degrees, slice thickness 2 $\mathrm{mm}$, FOV $150 \mathrm{~mm}$, scan matrix 256x256 and velocity sensitivity $30 \mathrm{~cm} / \mathrm{s}$.

The finite element package that was used in this study is called SEPRAN [10]. Application of the package for the analysis of cardiovascular flow has been carried out in collaboration with the Department of Biomedical Engineering at 
the Eindhoven University of Technology, the Netherlands [8]. The mesh used for the FEM-calculations consisted of triquadratic bricks with 27 nodes [12]. The curved tube could be defined with a small addition, namely the description of the curvature, within the mesh generator of SEPRAN. The bifurcation needed more additions, and had to be parameterized, but was also generated within the package. This is called the standard mesh.

The geometry of the carotid bifurcation was segmented from the MR Angiographic data set (figure 1) using the analytical software package MRA-CMS ${ }^{\circledR}$ [9] 1 . Each segmented vessel is expressed as a stack of circles, while a bifurcation is expressed by two stacks of circles (figure 2). The circles of the MRA-CMS ${ }^{\circledR}$ geometry were transformed to rectangles with the same spatial distribution as the rectangles at the surface of the standard mesh. A solver for linear elasticity was applied to transform the standard mesh [11]. The Young's modulus E was set at a value of 100 , while a small value was selected for the Poisson ratio $\left(\nu=1.0 e^{-4}\right)$ to allow for independent motion of the mesh in each coordinate direction. The standard mesh was thus transformed until it matched the MRA-CMS ${ }^{\circledR}$ data (figure 3).

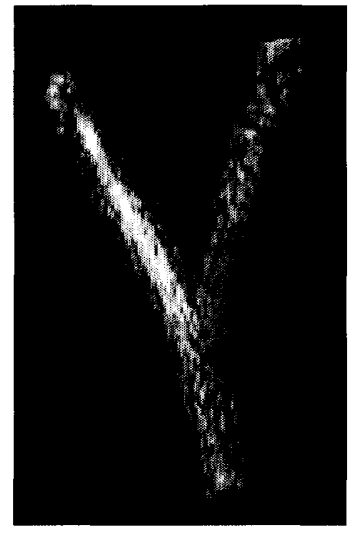

Fig. 1. Raw data from MRA

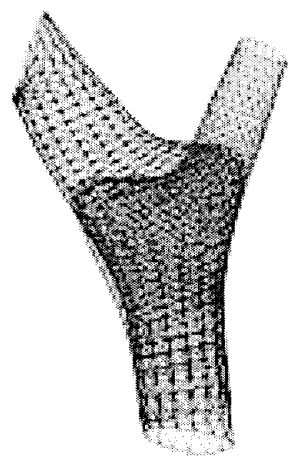

Fig. 2. Segmented carotid bifurcation phantom by MRA-CMS ${ }^{\circledR}$

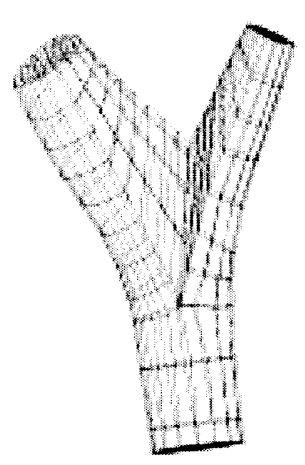

Fig. 3. Deformed mesh

The velocity encoded data were analyzed by means of the FLOW ${ }^{\circledR}$ analytical software package [3] ${ }^{1}$. FLOW ${ }^{\circledR}$ allows the quantification of the flow versus time and corresponding velocity profiles.

The flow values used as input for the calculations (flow $M_{R}$ ) were defined as follows: First, the velocity encoded data set was analyzed by means of the package FLOW $\left.{ }^{(}\right)$. The average velocity in the segmented area was calculated

\footnotetext{
${ }^{1}$ MEDIS medical imaging systems, Leiden, the Netherlands
} 
and multiplied by the surface of this area, yielding the volume flow value. Next, the flow in the curved tube was measured at three positions: at the entrance (inflow ${ }_{M R}$ ), in the middle $\left(\right.$ midflow $_{M R}$ ) and at the exit (outflow ${ }_{M R}$ ). Data of the carotid phantom were assessed at two positions, at the entrance of the flow (inflow ${ }_{M R}$ ) and just behind the bifurcation (intflow ${ }_{M R}$ and extflow $_{M R}$ ).

Flow ${ }_{M R}(\mathrm{t})$ was Fourier transformed and the first 20 Fourier Coefficients were used as input in the FEM calculations. After solving the Navier-Stokes equations, the flow (in the primary flow-direction) was calculated at the position where the MR-measurement was performed $\left(\right.$ flow $_{c a l c}$ ). Flow calc has to be put in the same grid as the MR-measurement. Therefore, a gridding and interpolation procedure was carried out. Then the flow and velocity profiles can be presented in the same manner as the MR-velocity measurements (flow image $_{\text {) }}$.

The calculation method was tested for the curved tube model under study at the Eindhoven University of Technology, the Netherlands [4]. The calculated flows and velocities were subsequently compared with the MRI-derived flow amounts and velocity profiles. For the purpose of comparing calculated with measured data [1], a special option was added to the standard FLOW ${ }^{\circledR}$ package. This option allows the assessment of the differences of calculated parameters and 2D velocity profiles in each time slice at each measured position. The 2-dimensional Kolmogorov Smirnov method was used for this purpose [7]. A measure was given for the difference between two distributions, when error bars (for individual data points) are unknown. Two semi-cumulative distributions of the two data sets under study were created. The maximum of the difference of the two distributions $\mathrm{D}_{K S}$ was taken (figure 4) and used for the calculation of $\operatorname{prob}_{K S}$.

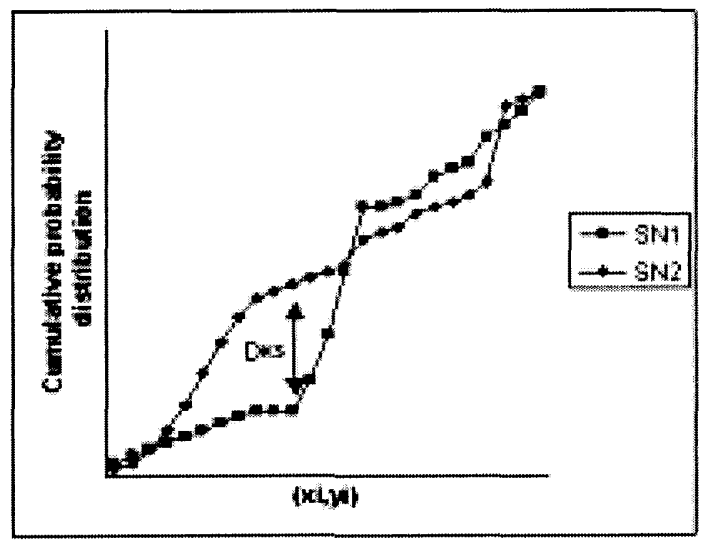

Fig. 4. $\mathrm{D}_{K S}$ gives the maximum difference between two arbitrary cumulative distributions SN1 and SN2 [6]. 
Prob $_{K S}$ gives the significance level of the comparison. If prob $K S$ is larger than 0.20 the two datasets are not statistically significant different. If $\operatorname{prob}_{K S}$ equals 1 , there is perfect correlation.

\section{Results}

The calculated flows and velocity profiles were compared with MR-measurements for the curved tube and the carotid phantom.

The results of the measurements and calculations of the curved tube are presented in figures 5 through 11 . In figure 5 flow $_{M R}$ is compared for the three

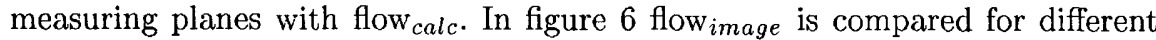
measuring planes to investigate the effect of gridding and interpolation. In figures 7 through 9 the velocity profiles at the three measuring planes are presented. The measurements and the simulations are plotted at the plane of symmetry for beginning, peak and end of systole, respectively (time slice nr 20,24 and 5). The results of the KS-test $\mathrm{D}_{K S}$ and $\operatorname{prob}_{K S}$ are presented in figure 10 and 11 , respectively.

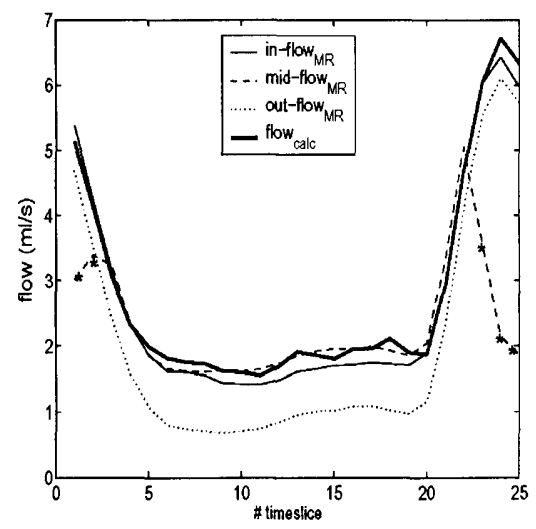

Fig. 5. Flow ${ }_{M R}$ and Flow calc $_{\text {for }}$ forflow, central and outflow region of the curved tube. * Indicates a phase-wrapping for some pixels in the measurement.

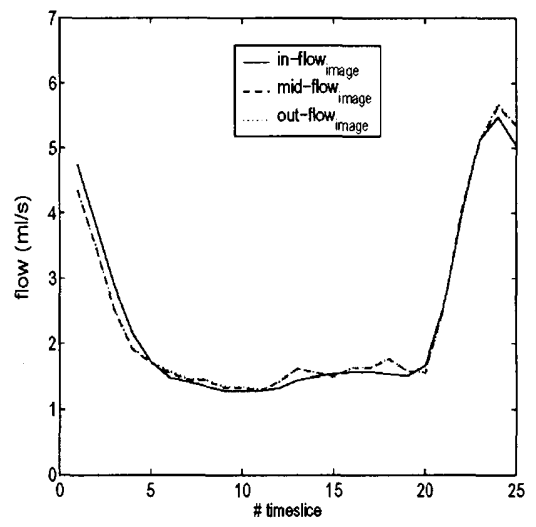

Fig. 6. Flowimage for inflow, central and outflow region of the curved tube.

For the carotid bifurcation the same procedure was used as for the curved tube. It has to be noted that some additional error sources are present here: 1) The outflow is stressfree, i.e. the probably present pressure difference between internal and external carotid is not taken into account; and 2) The MRA-CMS ${ }^{\circledR}$ package can segment single segments only. The segmented carotid bifurcation will therefore not exactly match the phantom (see figures 1 and 2) so the mesh 


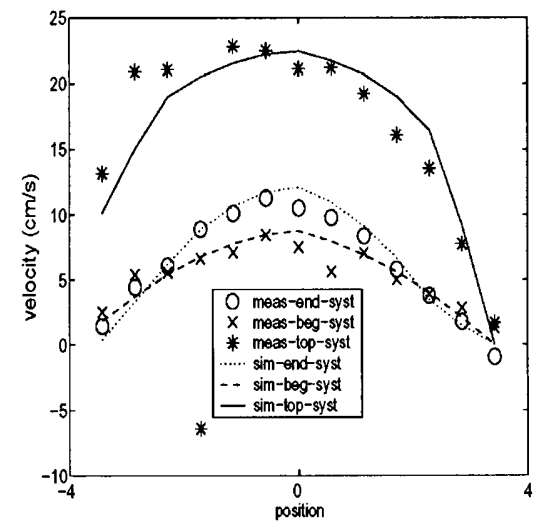

Fig. 7. Velocity profile in the plane of symmetry in the curved tube for inflow, at end systole, beginning of systole and peak systole

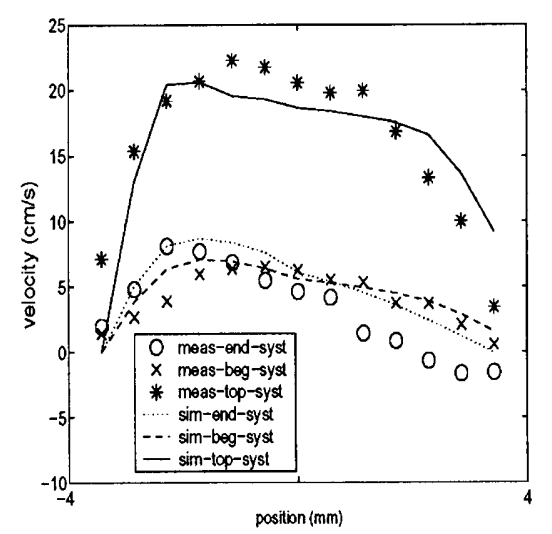

Fig. 9. Velocity profile in the plane of symmetry in the curved tube for outflow, at end systole, beginning of systole and peak systole.

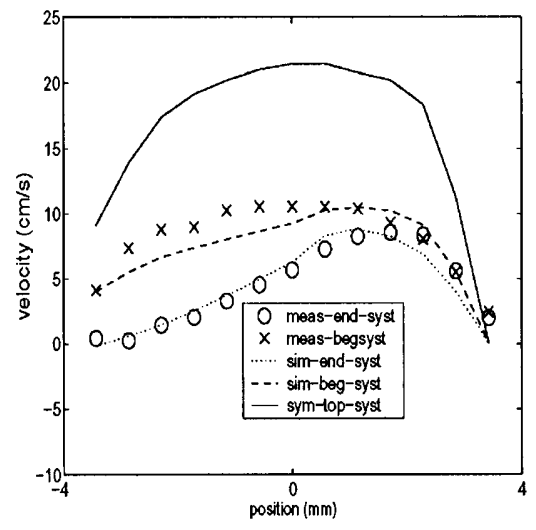

Fig. 8. Velocity profile in the plane of symmetry in the curved tube for the center of the bend, at end systole, beginning of systole and peak systole. Due to phase-wrapping errors the measurements at peak systole are not shown.

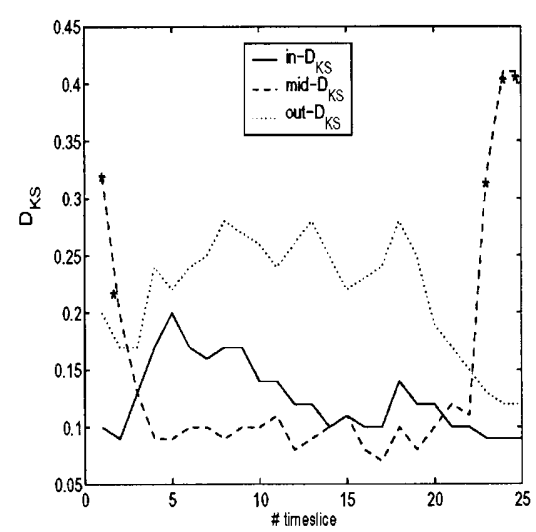

Fig. 10. $D_{K S}$ values for inflow, central and outflow region of the curved tube. * Indicates a phase-wrapping for some pixels in the measurement. 


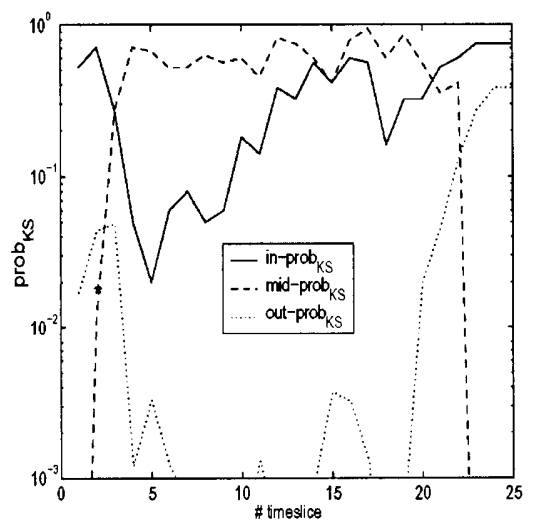

Fig. 11. prob $_{K S}$ values for inflow, central and outflow region of the curved tube. * Indicates a phase-wrapping for some pixels in the measurement.

cannot be optimal for the fluid dynamical calculations. For that reason, it is to be expected that the first results for the carotid bifurcation will not be as good as for the simple curved tube model. The carotid imaging planes were taken perpendicular to the inflow. The flow behind the bifurcation was split into two parts. One part for the external carotid and the other one for the internal carotid (see figures 12 and 13$)$. Prob $K S$ was very small $(\leq 0.001)$ everywhere for the carotid. Even at the in-flow (which is assumed to be a Womersley profile) these small values were measured.

\section{Discussion}

The curved tube shows a good similarity between MR-measurements and calculations in the middle of the bend when the errors due to phase wrapping are excluded. For the inflow and the middle of the bend prob $_{K S}$ is almost everywhere above 0.20 indicating that the difference between measurements and calculations is not significant. The outflow does not match the other measurements and calculations. The in-flow and central-flow were taken in the Feet-Head direction and the outflow in the Left-Right direction. Perhaps this is causing the non-optimal out-flow-measurements, which seem to be of good quality at visual inspection. Out-FLOW $_{M R}$ gives lower values in all the time slices and also out-Prob $K S$ indicates a poor similarity. In future research it will be inspected whether the direction of measurement has any effect on the results. Extra information for further analysis can probably be gathered from analysis of the secondary flows (vortices), which are also visible in the MR-data.

The carotid bifurcation demonstrates a difference between the calculations and the measurements as is illustrated in figure 13 and figure 12 . This is under- 


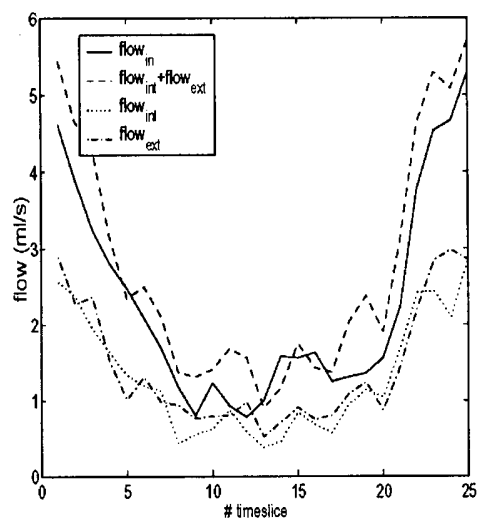

Fig. 12. Flow ${ }_{M R}$. In the figure Flow $_{i n}$ gives the inflow, and Flowint and Flow $_{\text {ext }}$ the flows in the internal and external carotid just behind the bifurcation respectively. Flow $i n t+$ Flow $_{i n t}$ gives the total Flow $M R$ measured behind the bifurcation.

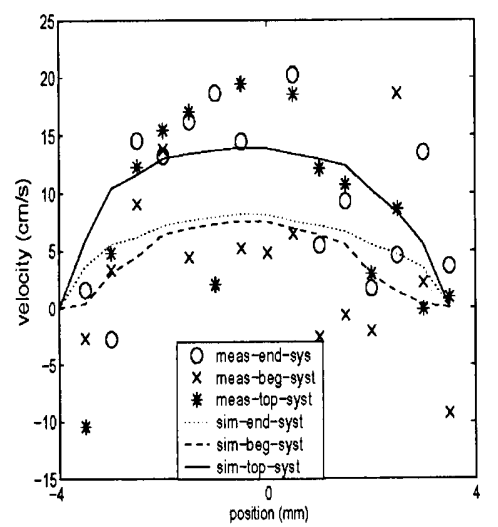

Fig. 14. The velocity of the inflow of the carotid is plotted for measurements and simulations for three time slices. For end of systole, beginning of systole and peak systole.

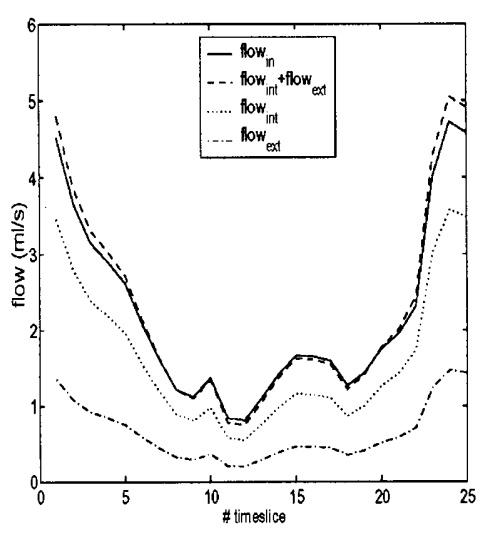

Fig. 13. Flow calc. In the figure Flow $_{i n}$ gives the inflow, and Flow int $_{\text {and }}$ Flowext gives the flow in the internal and external carotid just behind the bifuraction respectively. Flow Fint $_{+}+$ Flowext gives the total Flow culc $_{\text {calcu- }}$ lated behind the bifurcation. 
standable, because only the inflow conditions were pre-defined and no pressure or flow-profile for the outflow. The calculations for the carotid only indicate that the deformed mesh can be used for solving unsteady NS equations. For detailed error analysis additions have to be made in the near future. The MRmeasurements suffered from much more noise than the measurements of the curved tube, which is shown in figure 14. Therefore even at the inflow it is seen that prob $_{K S}$ is very small so that the KS data are not suitable. KS-analysis seems to be restricted to a certain noise level. With improved MRA bifurcation detection, measurements with less noise and prescribed outflow conditions, it is to be expected that KS-statistics in the carotid will become useable as well as for the curved tube.

\section{Conclusion}

The velocity profiles can be investigated in a quantitative manner with the KSstatistics and also be visualized individually. For the curved tube it was shown that the KS-statistics works well. Prob $K S$ is almost everywhere above 0.20 for the inflow and center regions, indicating that there is no statistically significant difference between measurements and calculations. The MRI flow measurements therefore are in good agreement with the calculated data. The computational method may be used to derive wall shear rates inside the chosen geometry $[8,4]$. With the proper viscosity model for blood, the wall shear stresses may also be computed using (2) [4]. For noisy measurements KS-statistics are not suitable. The mesh deformation algorithm works fine and the deformed mesh can be used for fluid dynamical calculations of carotids. In summary it can be concluded that flow $(\mathrm{t})$ and KS-results can indicate the amount of similarity between measurements and calculations. This approach opens the possibility for future in-vivo Wall Shear Stress measurements with MRI.

\section{References}

1. Box F.M.A., Spilt A., Van Buchem M.A., Reiber J.H.C., Van der Geest R.J.: Automatic model based contour detection and flow quantification of blood flow in small vessels with velocity encoded MRI. Proc. ISMRM 7, Philadelphia (1999), 571

2. Davies. P.F.: Flow-mediated endothelial mechanotransduction. Physiological Reviews, Vol 75, (1995) 519-560

3. Van der Geest R.J., Niezen R.A., Van der Wall E.E., de Roos A., Reiber J.H.C.: Automatic Measurements of Volume Flow in the Ascending Aorta Using MR Velocity Maps: Evaluation of Inter- and Intraobserver Variability in Healthy Volunteers. J. Comput. Assist. Tomogr. Vol. 22(6) (1998) 904-911

4. Gijsen, F.J.H., Allanic, E., Van de Vosse, F.N., Janssen, J.D.: The influence of non-Newtonian properties of blood on the flow in large arteries: unsteady flow in a 90 degrees curved tube. J. of Biomechanics. Vol.32(7), (1999) 705-713

5. Nichols W.W., O'Rourke M.F.: McDonald's Blood Flow in Arteries. Theoretical, experimental and clinical principles. Fourth edition. Oxford University Press, Inc. (1998) $36-40$ 
6. Press W.H., Teukolsky S.A., Vetterling W.T., Flannery. B.R.: Numerical Recipes in C. Cambridge University Press (1988), 491

7. Press W.H., Teukolsky S.A., Vetterling W.T., Flannery. B.R.: Numerical Recipes in C. Cambridge University Press (1992), 645-64

8. Rutten M.C.M.: Fluid-solid interaction in large arteries. Thesis Technical University Eindhoven, the Netherlands (1998)

9. Schaap J.A., De Koning P.J.H., Van der Geest R.J., Reiber J.H.C.: 3D Quantification and visualization of MRA. Proc. $15^{\text {th }}$ CARS (2000) 928-933

10. Segal G.: Ingenieursbureau SEPRA, Park Nabij 3, Leidschendam, the Netherlands

11. Johnson A., Tezduyar T.: Mesh update strategies in parallel finite element computations of flow problems with moving boundaries and interfaces. Computer methods in Applied Mechanics and Engineering, Vol 119, (1994) 73-94

12. Van de Vosse, F.N., Van Steenhoven, A.A., Segal, A., Janssen, J.D.: A finite element analysis of the steady laminar entrance flow in a 90 curve tube. Int. J. Num. Meth. In Fluids, Vol 9, (1989) 275-287

13. Womersley J.R.: An elastic tube theory of pulse transmission and oscillatory flow in mammalian arteries. Technical report, Wright Air Development Centre TR56-614, 1957. 